\title{
Les attentats de 1986 en France : un cas de violence transnationale et ses implications (Partie 1)
}

\section{Didier Bigo}

\section{(2) OpenEdition}

\section{Journals}

Édition électronique

URL : http://journals.openedition.org/conflits/129

DOI : $10.4000 /$ conflits. 129

ISSN : $1777-5345$

Éditeur :

CCLS - Centre d'études sur les conflits lilberté et sécurité, L'Harmattan

Édition imprimée

Date de publication : 17 janvier 1991

ISSN : 1157-996X

Référence électronique

Didier Bigo, «Les attentats de 1986 en France : un cas de violence transnationale et ses implications (Partie 1) », Cultures \& Conflits [En ligne], 04 | hiver 1991, mis en ligne le 31 décembre 2002, consulté le 30 mars 2021. URL : http://journals.openedition.org/conflits/129 ; DOI : https://doi.org/10.4000/ conflits. 129

Ce document a été généré automatiquement le 30 mars 2021

Creative Commons License 


\title{
Les attentats de 1986 en France : un cas de violence transnationale et ses implications (Partie 1)
}

\author{
Didier Bigo
}

Pour un universitaire, étudier le terrorisme, c'est accepter de se livrer au soupçon de certains de ses collègues. Le sujet est perçu, sinon comme un manquement à la déontologie professionnelle du moins comme une faute de goût. Pourquoi travailler sur le terrorisme quand existent tant d'autres sujets "propres"? L'universitaire en question devra accepter un interrogatoire en règle et on l'accusera vite de transformer la réflexion en investigations journalistiques ou pis encore d'être en collusion avec les services de police et les services secrets. Qu'il travaille sur les grèves, les manifestations, les émeutes, la répression, la guérilla ou la guerre, et le voilà blanchit ! L'illégitimité particulière du terrorisme se transmet-elle magiquement de l'objet d'étude à l'observateur de cette forme de violence politique ? On pourrait le croire. Et pourtant, à condition de prendre le recul nécessaire pour ne pas interpréter à chaud les événements, à condition aussi de ne pas accepter de se transformer en "expert" devant des médias avides de trouver des personnes se substituant aux policiers et aux magistrats lorsque ceux-ci refusent de parler ${ }^{1}$, il est tout à fait possible d'étudier cette forme originale de violence politique qu'est la relation terroriste ${ }^{2}$. En effet, contrairement à une idée reçue, les sources ouvertes, les possibilités d'entretiens avec les anciens acteurs, étant donné la rapidité du cycle des actions et leur faible ampleur, sont beaucoup plus grandes que pour des études sur la guérilla ou a fortiori la répression étatique. A propos des attentats qui se sont déroulés en France en 1985/1986, grâce aux documents des procès judiciaires, grâce aux différents témoignages de certains acteurs, on est en mesure quelques années après de connaître avec suffisamment de précisions les événements pour pouvoir proposer une grille de lecture explicative ${ }^{3}$.

\section{ELEMENTS D'HISTOIRE}

La série d'attentats et l'indétermination des services français 
Les attentats de décembre 1985 et la criminalisation:

A priori, dans la continuité des attentats du 23 février 1985 (magasin Marks \& Spencer : 1 mort, 14 blessés) ${ }^{4}$ et du 9 mars (cinéma Rivoli Beaubourg: 18 blessés), le double attentat des magasins "Galeries Lafayette" et "Printemps Haussmann", le 7 décembre (43 blessés), ouvre, semble-t-il, une nouvelle série d'attentats qui, en quatre vagues, frappera la France jusqu'en septembre $1986^{5}$. Mais, au départ, les enquêteurs ne pourront le deviner et s'orienteront vers deux pistes principales ; la première sera celle du groupe Abou Nidal en raison des soupçons pesant sur la responsabilité de ce groupe dans l'attentat Mark \& Spencer; la seconde, privilégiée officiellement, sera celle d'un acte commis par un déséquilibré comme dans les attentats du BHV. A l'appui de cette dernière thèse, les enquêteurs avançaient le caractère artisanal des engins incendiaires (bidons d'essence avec un peu de matière explosive et un petit détonateur actionné par un réveil), et une tentative identique l'année précédente au moment des fêtes de Noël. De plus, aucune revendication crédible n'était apportée sinon un coup de téléphone du Front de libération de la Palestine/groupe Abou Abbas et un autre de l'ASALA. Le juge Marsaud, chargé de l'enquête, était pourtant d'un avis différent et sceptique sur cette thèse de l'acte d'un fou, qui arrangeait les autorités. En effet, la matière explosive retrouvée était du C4 comme au Liban et l'on découvrait aussi des fragments d'un journal koweïtien $\mathrm{Al}$ Quabas qui enveloppait la machine incendiaire. L'hypothèse du Djihad islamique devenait alors crédible. Néanmoins, rien ne venait confirmer matériellement cette croyance. Il faudra attendre les attentats de février 1986 pour que l'hypothèse prenne corps.

Les attentats de février 1986 et la piste iranienne:

Le 3 février 1986, un attentat à la galerie marchande de l'Hôtel Claridge situé sur les Champs-Elysées, faisait 8 blessés et était revendiqué par le "Comité de solidarité avec les prisonniers politiques arabes et du Proche-Orient", ou "CSPPA". Le même jour, une tentative d'attentat par explosif était découverte dans les toilettes du troisième étage de la Tour Eiffel. Etait-ce un raté technique ou un avertissement? Les hypothèses se multiplieront, toujours est-il que l'on remarquera dans chaque série d'attentats au moins un raté; ce qui aura, outre l'absence de victimes, des conséquences positives pour l'enquête qui disposera de plus de matériaux techniques d'identification. Le lendemain, 4 février, une explosion ravageait la librairie Gibert Jeune faisant 5 blessés et le 5 février une autre frappait le magasin FNAC Sport du Forum des Halles faisant 22 blessés. Les deux attentats étaient aussi revendiqués par le CSPPA. Bien que l'on évoque au départ des vengeances d'employés pour la librairie Gibert, très vite cette fois l'enquête s'oriente vers les milieux chiites iraniens, tunisiens et libanais en France, ainsi que vers le Koweït peuplé de très nombreux Chiites iraniens (une commission rogatoire internationale est demandée à l'époque). Une série d'interpellations est ordonnée le 14 mars par le juge Marsaud à la suite de l'attentat de la galerie Claridge. Elle vise de nombreuses personnes appartenant au foyer Ahl El Beit, foyer qui sert de lieu de rendez-vous à l'intégrisme chiite, et qui fut dirigé par le frère de Cheikh Fadlallah ${ }^{6}$. Mais aucune charge ne peut être retenue contre les personnes interpellées qui seront libérées et expulsées. En effet, ce n'est que bien plus tard que l'on apprendra que l'on avait appréhendé en même temps les principaux responsables du réseau logistique des attentats et le poseur de bombe. Comme en décembre 85, les attentats sont commis avec des moyens artisanaux, poubelle piégée au Claridge... et sur la base d'un explosif, le $\mathrm{C} 4$, mélange d'octogène et d'hexogène. La DGSE révèlera qu'il s'agit du 
même explosif que celui qui avait été employé pour détruire une voiture devant l'ambassade de France au Liban le 12 décembre 1983. Le gouvernement français prend alors la mesure des événements et ne cherche plus, comme en décembre, à les dissimuler en les criminalisant. Il estime qu'il s'agit d'un message du Djihad essentiellement lié à la libération des membres de son organisation emprisonnés au Koweït, et dont on vient d'annoncer la condamnation à mort, ainsi qu'une pression supplémentaire sur la France pour qu'elle libère Anis Naccache, puisque le marchandage des otages français au Liban ne semble pas suffisant. La presse révèlera, quant à elle, que ce changement de politique gouvernementale tient au fait que le 5 janvier 1986 était prévue une libération de nos otages contre l'ensemble du commando Naccache et la libération des prisonniers du Koweït. Officiellement le gouvernement refusa la transaction dévoilée par la presse six mois plus tard car il considérait excessive les exigences du Hezbollah mais une thèse officieuse voudrait que l'opposition de droite de l'époque ait saboté la transaction en proposant d'accepter toutes les conditions lorsqu'elle arriverait au pouvoir. Elle y accède le 16 mars avec une très courte majorité à l'Assemblée Nationale. Le 17 mars les attentats recommencent.

Les attentats de mars 1986 et la piste Abdallah :

Le 17 mars, date de la nomination de Jacques Chirac comme Premier ministre, un attentat vise le TGV, alors qu'il circulait à hauteur de Brunoy, et fait 9 blessés. Le 20 mars, une bombe explose dans la galerie Point Show des Champs-Elysées faisant 2 morts dont Nabil Dagher fiché comme membre des FARL et 29 blessés. Le même jour, une tentative d'attentat est déjouée de justesse à la station Châtelet du RER, un des voyageurs étant intrigué par un paquet suspect. Une fois encore tous ces attentats, sauf ceux ratés, sont revendiqués par le CSPPA et le $\mathrm{C} 4$ entre dans leur composition. Sans indice, la police et le nouveau gouvernement français restent impuissants devant la troisième vague d'attentats de 1986, d'autant qu'immédiatement au pouvoir, ils multiplient les contacts avec les ravisseurs des otages, font des concessions et croient que les Iraniens leur en sont reconnaissants ${ }^{7}$. Dès lors l'enquête s'oriente vers d'autres pistes délaissées et privilégie cette fois l'hypothèse du clan Abdallah dont le frère Georges Ibrahim est sous les verrous de la justice française malgré des promesses officieuses de libération en contrepartie du fils de Gilles Peyrolles relâché par les FARL. Persuadé que la famille Abdallah, flouée par ce marché de dupes ${ }^{8}$, est derrière ces attentats, le gouvernement prend des contacts avec les Algériens et les Syriens pour qu'ils calment les FARL et les surveillent. Or, à partir de cette date, une accalmie semble enfin se produire, comme si la commission des attentats avait partie liée avec les péripéties du procès de Georges Ibrahim Abdallah.

Les attentats de septembre 1986 et la piste syro/abdalienne:

Du mois d'avril au mois d'août, le nouveau gouvernement français respire. Il pense avoir trouvé la solution pour prévenir les attentats à Paris en faisant surveiller les Abdallah par les Syriens. Aussi, la quatrième vague d'attentats, de loin la plus meurtrière, le prendra-t-elle totalement au dépourvu et provoquera-t-elle le sentiment d'avoir été trahi par la Syrie. En effet, l'organisation ou les organisations qui s'abritent derrière le sigle CSPPA, recommencent à frapper Paris comme jamais auparavant. La série s'ouvre par un attentat raté dans le RER à la station Gare de Lyon le 4 septembre. Quatre jours plus tard, le 8 septembre, un attentat vise le bureau de poste de l'Hôtel de ville de Paris faisant 1 mort et 21 blessés. Quatre jours après, le 12 septembre, un attentat frappe les locaux de la Cafétéria Casino au Centre commercial de la Défense et 
fait 54 blessés. La série s'intensifie encore avec des dates plus rapprochées. Le 14 septembre, un attentat est déjoué au Pub Renault sur les Champs-Elysées mais 2 policiers et 1 serveur sont tués et un autre serveur blessé par l'explosion de l'engin déplacé. L'attentat, sinon, aurait fait beaucoup plus de victimes. Le 15 septembre, un attentat vise les locaux du Service des permis de conduire de la préfecture de police à Paris faisant 1 mort et 56 blessés. Enfin le 17 septembre, l'attentat commis devant le magasin Tati, rue de Rennes clos la série, et se révèle le plus meurtrier de tous : 7 morts et 55 blessés. Tous ces attentats sont revendiqués par le CSPPA et par un groupe qui s'intitule "Partisans du droit et de la liberté". Le gouvernement effaré par la violence de ces attaques ne sait plus que penser et a du mal à imaginer qu'une famille libanaise puisse, pour faire libérer un frère emprisonné, aller jusque-là. On suppose très rapidement qu'il a fallu des soutiens étatiques importants pour cette campagne d'attentats et toutes les pistes redeviennent ouvertes: action iranienne par l'intermédiaire du Hezbollah, vengeance des frères Abdallah plus ou moins aidés par d'autres organisations comme l'ASALA, action syrienne par l'intermédiaire des FARL... Cette dernière hypothèse sera privilégiée tant dans les médias que dans les discours des premiers jours du gouvernement français, où le langage guerrier fait des ravages. Seulement, face aux protestations syriennes, le gouvernement revient très vite à la thèse des frères Abdallah sans soutien syrien, au grand déplaisir des médias, et se tiendra à cette thèse jusqu'à la découverte des véritables responsables de tous ces attentats : le réseau Ali Fouad Saleh ${ }^{9}$.

Les auteurs des attentats

La nouvelle série prévue en 1987 :

Peu après la condamnation à perpétuité de Georges Ibrahim Abdallah, une nouvelle série d'attentats était prévue. Elle devait ensanglanter à nouveau Paris. A cet effet, le responsable du groupe Ali Fouad Saleh devait recevoir de Mohamed Ali Hamade, en Allemagne, du nitrate de méthyle, explosif liquide très instable, mais très puissant. Etant donné l'importance des stocks retrouvés, la série prévue aurait été sans doute bien plus longue et bien plus sanglante que celle de septembre ${ }^{10}$. Mais la DST réussira à interpeller les coupables avant qu'ils ne commettent de nouveaux attentats et elle démantèlera le réseau logistique de l'organisation travaillant en France en retrouvant quasiment toutes leurs caches. Grâce à ce succès dont la police et le gouvernement avaient bien besoin, on sut mieux comment les autres attentats avaient été réalisés, puisque, parmi les personnes interpellées, certaines admirent que leur groupe était responsable sur le plan logistique de l'ensemble des opérations menées sur le territoire français depuis décembre 1985. Pourtant la presse, si elle applaudit à l'arrestation des coupables, ne revint pas sur les interprétations erronés qui l'avait guidée depuis plus d'un an (mars 1986 - juin 1987) et, finalement, on se rend compte que l'information sur les véritables coupables des attentats a été bien moins importante que celle sur les fausses pistes ${ }^{11}$. Au départ, la découverte du groupe Ali Fouad Saleh repose sur deux indices différents qui se recouperont. Tout d'abord, une des personnes interpellée en février 1986, nommée Lofti Ben Khala, et plus ou moins suivie par les Renseignements généraux depuis, vint se présenter en février 1987 pour proposer des renseignements sur les attentats de septembre, moyennant finances. Lofti aurait été en contact avec les Iraniens (Rechari et Rafigh Doust) à Téhéran. Ce dernier aurait parlé d'un "plan" élaboré par les Iraniens pour frapper la France en réponse au prêt par celle-ci des Super étendards à l'Irak afin de détruire le terminal pétrolier iranien de Kharg, et aurait fourni un document iranien faisant l'éloge des actions clandestines menées à Paris par 
le Hezbollah ${ }^{12}$. Lofti, revenu en France, et fort de ses contacts iraniens, réussit alors à s'infiltrer au sein du réseau clandestin et désigne à l'attention des services de police un groupe d'individus qui avait un appartement au 44 bis rue de la Voute dans le 12ème arrondissement, appartement qui était officiellement une école coranique. La DST pose des écoutes téléphoniques et des micros et le 21 mars 1987, apprend que les membres du réseau vont déplacer les explosifs stockés dans l'appartement. Une discussion s'en suit pour savoir s'il faut remonter la filière le plus longtemps possible ou les arrêter compte tenu du risque qu'ils représentent. M. Pandraud choisit cette dernière solution et la DST arrête les principaux responsables du groupe Ali Fouad Saleh, c'est-à-dire luimême, un employé de restaurant Mohamed Aïssa et un chauffeur de taxi, Hassan Aroua. Dans le flagrant délit on trouve des bouteilles d'explosif liquide identiques dans leur conditionnement à celui du groupe Hamade en Allemagne. Le lendemain les interpellations se poursuivent: On arrête la femme d'Ali Fouad Saleh, Karima Fehari ; Mohamed Mouhajer, ex-responsable du Centre culturel iranien de la rue Jean Bart, fermé en 1983 et libraire depuis ${ }^{13}$; un gérant de société de parfums, Fetih Bourguiba, ami d'Ali Fouad Saleh, (plus deux autres personnes qui seront très vite relâchées). Hassan Aroua et Fetih Bourguiba, dès le premier jour de l'arrestation, chargeront Ali Fouad Saleh et reconnaîtront le rôle du groupe dans les attentats de septembre. Ils indiqueront aussi à la police le nom de code de plusieurs Libanais qui auraient, eux, commis directement les attentats puis seraient repartis. Parallèlement à ces événements, les services français recevaient de leurs homologues ouest-allemands, en janvier 1987, des renseignements concernant les liens qui existeraient entre le groupe Hamade arrêté en RFA le 13 janvier pour détention d'explosifs et le groupe français ${ }^{14}$. Selon le Bundeskriminalant ${ }^{15}$ les explosifs d'Hamade étaient destinés au groupe parisien après un passage par Frankfort et auparavant par Chypre et le Liban. Le groupe Hamade en RFA avait en effet comme mission, semble-t-il, d'approvisionner en armes et en argent les autres groupes européens et l'on retrouvera plusieurs numéros de téléphone du groupe Fouad Saleh chez Hamade: tout d'abord celui de Ali Fouad Saleh lui-même sous le pseudonyme d'Ali el Tounsy (le Tunisien), ensuite celui de Mehdi Diab à l'adresse de Fetih Bourguiba, et, enfin, celui d'Hussein Mazbou sous la fausse identité qu'il employa à plusieurs reprises Hassan Ali. De plus le conditionnement des explosifs, les sacs servant à les transporter et les modalités de falsification des passeports sont identiques dans les deux groupes. A ce stade, il est impossible de croire à des coïncidences. Les deux groupes Hamade et Fouad Saleh coopéraient étroitement. Le groupe Hamade convoyant les explosifs (du nitrate de méthyle) jusqu'en France et de la drogue soit pour s'autofinancer, soit parce qu'un des frères Hamade au Liban dirige une filière de drogue.

Les arrestations du groupe Fouad Saleh :

Après l'arrestation du 22 mars, les policiers vont remonter la filière tant sur le plan des autres membres du groupe Fouad Saleh que sur celui des caches d'explosifs. Tout d'abord, malgré le mutisme obstiné de Fouad Saleh, ils reconstituent son itinéraire. Celui-ci, né à Paris le 10 mai 1958, de nationalité tunisienne, a passé toute son enfance à Tunis qu'il ne quittera que pour l'Iran en 1982. Après un bref séjour à Qom, il revient à Tunis d'où il repart immédiatement pour Paris. Très lié au renouveau coranique de la capitale et à la création de nouvelles mosquées, il se lie d'amitié avec Mohamed Mouhajer, étudiant libanais en philosophie à la Sorbonne qui a suivi, lui aussi, des cours à l'université de Qom en Iran. Ce dernier l'aide à repartir à Qom pour un séjour plus long et lui fait rencontrer à cet effet Vahid Gordji, attaché à l'ambassade d'Iran. Vahid 
Gordji obtiendra pour Fouad Saleh une bourse d'études pour Qom où il restera un an. Là-bas ces rapports avec les autorités iraniennes sont sujet à controverse, toujours estil qu'officiellement Ali Fouad Saleh est emprisonné à la mi-1984, puis expulsé vers la France ${ }^{16}$. Il y arrive en mai ou septembre. Devant l'esplanade du centre Beaubourg où il se lance dans la prédication, il rencontre Mohamed Mehdi, "combattant" du Hezbollah libanais. Mohamed Mehdi revient entouré d'autres de ses amis pour écouter Fouad Saleh dont l'ardeur dans les prêches attire un nombre important de personnes. Il lui fait rencontrer Alaa Alaeddine, Sami slim et d'autres membres du Hezbollah. Parallèlement, Fouad Saleh retrouve Abdelhamid Badaoui, étudiant marocain qu'il avait connu en 1982 et lui présente certaines de ses relations, tunisiens comme lui : Aïssa et Aroua. S'agissait-il dès cette époque pour lui de recruter sur ordre du Hezbollah ou ces derniers étaient-ils dans l'ignorance des attaches de Fouad Saleh avec les Libanais et le prenaient-ils simplement pour un homme inspiré par la foi religieuse ? Il est difficile de trancher. Plusieurs membres du groupe, interpellés par la police, se défendront de connaître exactement les activités de Fouad Saleh, et auraient entreposé ses affaires sans savoir ce qu'elles contenaient. Ils auraient voulu aider un passionné de Dieu, sans logement, vivant en vendant des fruits dans le métro et n'auraient pas compris qu'Ali Fouad Saleh se servait de leur hospitalité pour entreposer chez eux des explosifs ${ }^{17}$. Toujours est-il que la délation interne entre les membres du réseau va prendre un tour extraordinaire et va permettre à l'enquête de progresser rapidement. Grâce à l'étude du carnet d'adresses de Fouad Saleh et aux déclarations d'Aïssa et d'Aroua qui, immédiatement, chargent Fouad Saleh et se disent innocents malgré le flagrant délit, les policiers pourront interpeller à nouveau plusieurs personnes: Tout d'abord, Omar Agnaou et Abdelhamid Badaoui, tous les deux Marocains. Badaoui accepte de parler s'il peut bénéficier de la loi des "repentis" et va encore plus loin que ceux qui s'étaient dissociés. Il affirmera avoir été trompé par Fouad Saleh au départ puis avoir pris peur des conséquences ensuite lorsqu'en septembre 1986, il aurait compris enfin que son ami était à la tête de l'organisation qui commettait les attentats à Paris. Il se dit "soulagé" et accepte de témoigner contre les autres accusés. Il donne ainsi le nom de code d'un des opérationnels venu du Liban, Bassam, et révèle une cache dans la forêt de Fontainebleau. Peut-on pour autant croire à la bonne foi de Badaoui et n'y voir qu'un individu trop confiant manipulé par le réseau? Difficile. Badaoui a entreposé chez lui, outre les sacs ayant servi au transport du $\mathrm{C} 4$, des connecteurs de piles identiques à ceux des attentats ratés et surtout il reconnaîtra s'être défaussé des sacs d'explosifs au profit d'Agnaou car il ne voulait pas garder ces sacs chez lui ! Agnaou reconnaîtra avoir eu les sac d'explosifs et confondra Badaoui en assurant que ce dernier l'avait prévenu de leur nature dangereuse. La forte délation des membres du groupe entre eux change l'image que la presse a voulu donner du réseau Fouad Saleh. Contrairement à une idée reçue et amplifiée par les médias, braqués exclusivement sur les imprécations d'Ali Fouad Saleh, le groupe n'est pas soudé par une croyance dans la vertu de la Révolution iranienne et par le chiisme. Il ne s'agit nullement, au cas près de Fouad Saleh, de "fanatiques". Leur foi, si elle existe, n'a rien d'exaltée. Plusieurs ne sont guère pratiquants et consomment de l'alcool... Ils sont de confessions différentes : chiites et sunnites. L'examen précis du dossier met à mal l'image d'un groupuscule animé par la foi religieuse et prêt à se sacrifier à la Cause. La faible solidarité du groupe fait d'ailleurs hésiter sur le vocabulaire. Seul semble-t-il Fouad Saleh est convaincu de la justesse de la cause qu'il défend, les autres, complices, l'aident mais feignent de ne pas comprendre la portée de leurs actes. Tout leur système de défense reposera sur ce point. Nous sommes loin des 
procès politiques et militants. S'agit-il même d'une organisation structurée ou d'un réseau lâche de relations? A part Fouad Saleh, qui agit par conviction? A priori, personne dans le groupe des individus interpellés de nationalité tunisienne et marocaine ${ }^{18}$. A cet égard, l'attitude des Maghrébins recrutés par Fouad Saleh contraste avec celle des six Libanais qui seront interpellés. Refusant de reconnaître une quelconque participation aux attentats, les Libanais (Alaa Alaeddine, Anwar Jomaa, Nasser Reda Hachem, Farid Roumi, Tashin Saad et Sami Slim) auront un système de défense plus serré et admettront simplement avoir rencontré dans diverses occasions Ali Fouad Saleh. Plusieurs d'entre eux fréquentent le foyer Ahl El Beit, appartiennent au Hezbollah et ils sont sans doute plus au coeur du réseau clandestin que les membres marocains et tunisiens arrêtés. Pourtant la presse, à l'époque de leur arrestation comme lors du procès, les laissera dans l'ombre car les charges retenues contre eux sont très légères et qu'ils apparaissent à beaucoup comme de simples comparses. En effet, la presse insiste sur la nationalité maghrébinede certains membres du réseau pour dresser un tableau pour le moins ambigu des rapports entre terrorisme et immigration. Elle fantasme sur les communautés relais implantées en France par les Etats étrangers et un certain nombre de "spécialistes" du terrorisme lui emboîtent le pas ou la précèdent ${ }^{19}$. On cherche à persuader l'opinion que le réseau Fouad Saleh vit comme un poisson dans l'eau au sein des immigrés et que c'est là qu'il recrute. Les manchettes de journaux sur l'intégrisme se multiplient... Pourtant, là aussi une lecture attentive des documents montre à quel point il fut difficile à Fouad Saleh de recruter et comment les agents opérationnels durent venir du Liban. En exagérant, on pourrait presque dire que la compréhension véritable du réseau Fouad Saleh passe par l'idée qu'il fut justement impossible de recruter dans l'immigration française car celle-ci n'adhérait en rien, même chez les plus radicaux, au projet d'une "sale guerre" contre les Français ${ }^{20}$. Fouad Saleh n'a pu convaincre que quelques proches et encore en leur cachant en grande partie la vérité. Le Hezbollah a dû faire appel à des membres de son organisation à Paris et à des membres venus du Liban pour commettre les attentats. Il y a altérité des réseaux sociétaux, transfert quasi-complet d'hommes et de matériels sans réelle infrastructure sur place, ce qui explique en grande partie les faiblesses du réseau. Celui-ci n'arrive pas à s'internaliser, à mordre sur l'immigration en France.

Les indices concernant la participation d'auteurs libanais :

D'après ce que l'on peut savoir, l'équipe maghrébine de Fouad Saleh n'avait donc qu'un rôle de soutien logistique, elle n'a ni déposé les explosifs, ce qui serait le fait de plusieurs Libanais venus puis repartis immédiatement dans tous les cas, ni écrit les revendications du CSPPA ou du PDL, ce qui serait le fait d'une troisième équipe restée à Beyrouth et d'un seul scripteur à Paris qu'aucune investigation ne permettra de trouver $^{21}$. Tout ce que l'on sait du groupe libanais se réduit à peu de renseignements tirés des confessions des "repentis" : six noms (Nizar El Zein, Ibrahim Akil dit Tashin ou Ali Ghosn, Mohamed Mehdi Diab dit Abou Amer, Ali Hassan, Hussein Mazbou et Haïdar Habib dit Bassam ou Nasredin ou Nasser Eddine) et un numéro de téléphone au Liban renvoyant à un quartier contrôlé par le Hezbollah, où les otages français passèrent une partie de leur détention ${ }^{22}$. Informations vérifiées par la police de l'air et des frontières qui, effectivement, retrouva ces noms dans des vols précédant de peu les principales séries d'attentats. De plus, on sait par Badaoui qu'Habib Haïdar, l'artificier du groupe libanais, vint chez lui chercher des explosifs au moins à deux reprises en mars, puis en septembre, après que, le 15 juin, Fouad Saleh ait ramené un nouveau chargement. Seulement, de nombreux points obscurs subsistent: quels étaient les liens entre les 
deux équipes, la libanaise opérationnelle et l'équipe logistique Fouad Saleh ? A part Fouad Saleh, y avait-il d'autres contacts en France pour les poseurs de bombe ? Quels étaient les liens entre l'équipe Fouad Saleh et l'équipe Hamade ? Comment ont transité les explosifs, les armes, l'argent et les hommes nécessaires à la commission des attentats? Les poseurs de bombes étaient-ils des mercenaires payés ou des individus idéologiquement motivés et appartenant au Hezbollah? De qui recevaient-ils leurs ordres et quelle marge de manoeuvre avaient-ils ? Ont-ils choisi eux-mêmes les cibles, ou leur a-t-on dit où frapper? Plus "professionnelle", l'équipe libanaise, si tant est qu'elle ne se réduit pas à un homme, semble avoir fait attention à ne pas être trop en contact avec d'autres personnes que Fouad Saleh, même si certains durent rencontrer Badaoui et Agnaou pour récupérer les explosifs cachés chez eux. On peut d'une certaine manière distinguer deux périodes: les attentats de décembre 85 et février 86 dont le maitre d'oeuvre serait Hussein Mazbou et après l'expulsion de celui-ci, les attentats de mars et septembre 86 dont l'artificier serait Habib Haïdar dit Bassam. Hussein Mazbou, haut responsable du Hezbollah et un des chefs de leur service secret serait venu à Paris en mai 1985 où il aurait remis une valise d'explosifs à Fouad Saleh qui l'aurait déposée chez Badaoui. Au mois d'octobre Ibrahim Akil, alias Ali Ghosn rejoignait Mazbou à Paris en compagnie d'Hassan Ghosn. Il est vraisemblable que les attentats de décembre 85 furent réalisés par cette équipe, en liaison avec quelques uns des Libanais cités plus haut et peut-être Ali Fouad Saleh. L'absence de crédibilité des premières revendications laisse penser à une phase de "test" avant de se lancer dans la campagne de février. Hussein Mazbou devait en effet revenir fin janvier à Paris pour programmer l'ensemble des attentats et en faire une "campagne" programmée sur plusieurs mois, mais le réseau était désorganisé par l'expulsion de Mazbou en février. On envoyait alors à la place de Mazbou, Habib Haïdar, membre plus obscur du Hezbollah, parlant mal le français..., pour prendre la place de responsable des opérations et d'artificier du réseau. Le rôle d'Ali Fouad Saleh fut alors sans doute plus important car il devait assumer une partie des tâches qui auparavant incombait à Mazbou. C'est à cette époque semble-t-il qu'on lui donna le numéro de téléphone du quartier général du Hezbollah. Fouad Saleh accompagna Habib Haïdar chez Badaoui, le présentant sous le nom de Bassam et demanda à Badaoui de le laisser utiliser la valise. Aïdar repartira de Paris après le 20 mars, et les attentats cesseront. Pourquoi cet arrêt? S'agit-il de laisser le temps au nouveau pouvoir français de négocier après lui avoir rappelé qu'il serait aussi la cible d'attentats? Peut-être, même si l'on peut être surpris que le Hezbollah brouille en même temps les pistes. Une rationalité moins stratégique et plus prosaïque tient peut être à l'absence de plan programmé plus avant et à la nécessité d'en référer à Beyrouth. Comme en juillet et août, Badaoui et Agnaou s'en vont en vacances, les opérations sont repoussées à la rentrée ${ }^{23}$. Haïdar revient donc simplement au mois de septembre pour la nouvelle série d'attentats. Hormis Haïdar, combien étaient les Libanais venus du Liban? Etaient-ils en contact avec les Libanais de Paris ou simplement avec Fouad Saleh ? Il est difficile de juger. Il semble en tout cas que chaque poseur de bombe ait été un (ou plusieurs) membre libanais actif du Hezbollah, agissant sur le territoire français et en repartant immédiatement, laissant le soin à Fouad Saleh de faire les repérages. La connexion avec le Hezbollah est donc quasiment certaine. C'est cette organisation qui a programmé les attentats en France en s'appuyant sur un réseau volant très limité (une ou deux personnes) et sur un réseau sur place lui aussi très réduit et dont l'allégeance tient à la seule personne de Fouad Saleh. 
Les réseaux transfrontières mis au point par le Hezbollah : manque de moyens, amateurisme... mais efficacité malgré tout.

Il est délicat de se prononcer avec certitude sur les liens avec la direction officielle du Hezbollah libanais faute de preuve judiciaire mais les éléments réunis lors des procès français et allemand sont importants. Hussein Mazbou, Ibrahim Akil, Mohamed Mehdi, Hassan Ghosn, Abdelahdi Hamade ${ }^{24}$ sont autant de responsables importants du Hezbollah. Ils entourent Igmad Moughnieh et cheikh Fadlallah. Ils sont à la tête de familles lourdement impliquées dans les prises d'otages occidentaux à Beyrouth et dans les détournements d'avions qui y sont liés. Aussi peut-on penser que les hommes entraînés à commettre des attentats, la planification de ceux-ci, l'origine des explosifs et des armes proviennent de la direction du Hezbollah. Les poseurs de bombes, appartenant à des familles chiites connues pour leur appartenance au Hezbollah ou en étant très proches, sont venus à Paris avec des passeports libanais en bonne et due forme si l'on excepte le changement de photographie, et cela ne fait que mettre en lumière la faiblesse à l'époque des services de renseignements extérieurs français. Ils sont passés à travers les contrôles de frontières à plusieurs reprises sans véritablement être inquiétés si ce n'est Abas Hamade ${ }^{25}$ en 1987. Les explosifs, quant à eux, ont suivi différentes filières selon les moments mais les grandes lignes sont identiques. On ne connait pas la manière dont ils sont passés en 1985 et comment la valise d'une trentaine de kilos de $\mathrm{C} 4$ a été convoyée. En revanche, on est particulièrement bien renseigné sur les transits d'explosifs qui eurent lieu fin 1986 et début 1987 et qui contribuèrent à faire chuter le réseau. Tout d'abord il faut rappeler que les déclarations de Badaoui lors du procès ont permis de comprendre pourquoi les attentats s'étaient arrêtés après la rue de Renne en septembre 1986. On s'était beaucoup interrogé en supputant des accords secrets entre le gouvernement français et les poseurs de bombes..., la raison plus prosaïque fut que le réseau n'avait plus d'explosifs et que Fouad Saleh et Haïdar Habib furent obligés de partir à Beyrouth pour rendre compte de leurs actions et pour en recommander. Les nouveaux stocks d'explosifs qui arriveront à partir d'octobre 1986 ne sont plus composés de C4 mais, d'une part de pentrite et d'autre part de nitrate de méthyl. L'importation de l'explosif solide (la pentrite) se fera par le biais de rouleaux d'imprimerie dans lesquels on masquera l'explosif. Le 30 septembre 1986, un premier colis d'une soixantaine de kilos part de Beyrouth expédié par la société AKS (Abdul Karim, Hussein Jaafar) via le transitaire C/Itoh dirigé par Tony Daoud. Il est envoyé en France à une société franco-libanaise : la CSC dirigée par Sadek Kronfol. Ce dernier, dont la société est en liquidation judiciaire fait affaire habituellement avec l'entreprise Nassar dont le gendre est Toni Daoud. Choisi en fonction de liens personnels des membres du réseau plus qu'en raison d'une couverture officielle solide (la société est en liquidation judiciaire), Kronfol fait récupérer à Orly frêt le 22 octobre les deux colis du premier arrivage et les entrepose dans sa société, avenue des champs-Elysées. Il les stocke en attendant qu'Habib Haïdar vienne les chercher sous le nom d'Abbas Nasredine le 25 novembre. Un deuxième colis partira de Beyrouth le 9 novembre en suivant le même trajet mais la lettre de transport aérien n'arrive pas à Kronfol et le colis est stocké dans les entrepôts de la Chambre de commerce à Orly le 21 novembre. Kronfol téléphone à la société France Handling afin qu'ils récupèrent le colis le 8 décembre et qu'ils le gardent en attendant que la société DEFIE leur dédouane. Cette société le fait le 18 décembre. Haïdar essaie le 18 d'aller chercher les colis mais n'ayant pas de lettre de transport aérien, il s'en voit refuser l'accès. Il téléphone à Kronfol qui lui en remet une et le 19 décembre le deuxième colis 
est récupéré par Haïdar et Aroua, ce dernier devant mettre de sa poche pour payer la facture car Haïdar n'a pas pris assez d'argent sur lui. Les explosifs sont alors convoyés jusque chez Bourguiba. Là les colis sont découpés et l'explosif est réparti dans des sacs afin d'être enterrés dans des poubelles qui ont été préalablement enfouies en forêt de Fontainebleau au début du mois. Ces poubelles seront retrouvées par la police scientifique de la DST après les indications de Badaoui et Aroua. Le 26 mai 1987, une première poubelle scellée avec du goudron et contenant $8,79 \mathrm{~kg}$ d'explosif et $6,5 \mathrm{~kg}$ d'héroïne, mélangés à de la caféine sera retrouvée par les équipes de police. Une seconde, vide, le sera le 29 décembre. Elle contenait, après examen, des traces de tolite, l'explosif utilisé contre le bureau de poste de l'Hôtel de ville et le pub Renault. L'importation du nitrate de méthyle se fera via l'Allemagne par l'équipe Hamade. Les deux frères Hamade se chargeaient du conditionnement du nitrate de méthyle dans des bouteilles d'Arak et les acheminaient du Liban en Allemagne, en Sarre, à la frontière française, via l'aéroport de Larnaca. Là elles étaient convoyées semble-t-il par deux hommes, Youssef Mechref et Mohamed Mehdi Diab, vers la France et remises à Fouad Saleh $^{26}$. Il est impossible de ne pas s'interroger sur le côté artisanal des transferts d'explosifs. Nous sommes très loin de l'organisation de groupes comme le FPLP ou Abou Nidal. Nous le sommes encore plus d'un groupe soutenu par un Etat et qui pourrait user de la valise diplomatique pour convoyer les explosifs. Il résulte de cette description une impression d'amateurisme, de "trafic" qui renvoie beaucoup plus aux techniques habituelles des réseaux transnationaux de la diaspora libanaise qu'à des pratiques étatiques ${ }^{27}$. Mais cet amateurisme a ses avantages, il se perd dans la foule des "petites combines" habituelles au milieu et n'éveille pas l'attention des services de police qui, en revanche doivent surveiller de près les allées et venues des "diplomates" du MoyenOrient. Bref on voit à quel point les pratiques mercantiles habituelles du Liban, fortement liées au clientélisme, à l'interconnaissance, à l'absence de règles juridiques, au paiement en liquide, aux "arrangements", moulent les comportements des membres $\mathrm{du}$ réseau libanais. Ainsi, les logiques transnationales d'acteurs subétatiques (libres de souveraineté) n'en sont pas moins plus difficiles à détecter pour des Etats que les techniques habituelles d'infiltration de services secrets adverses. Ces éléments matériels remettent en cause l'idée d'un soutien actif des Iraniens aux attentats de 86. On peut même penser que c'est la croyance en ce soutien qui explique les erreurs d'analyse (donc de surveillance) qui ont été faites à l'époque et qui se prolongent actuellement dans les milieux policiers et judiciaires. Croyant à une "affaire d'Etats", ils ne peuvent en effet raisonner que dans le cadre de l'interétatique, des relations internationales classiques et ne sont pas à même de penser l'interpénétration des sphères transnationales et étatiques, caractéristique de notre époque. Cela les amène sans doute à surévaluer le rôle des Iraniens en voyant en eux un gouvernement homogène, rationnel, chef d'orchestre clandestin d'une stratégie indirecte, bref en lisant les actions des organisations, des communautés et des factions moyen-orientales par le prisme déformant de la thèse de l'internationale terroriste $2^{28}$

Les indices concernant la participation directe des Iraniens dans les attentats :

Avec la découverte du réseau Fouad Saleh/Hamade, remontant jusqu'au Hezbollah libanais, la piste d'une participation iranienne directe aux attentats fut relancée. Le gouvernement comme les médias furent persuadés que l'Iran parrainait les opérations Fouad Saleh Hamade par l'intermédiaire du Hezbollah et même qu'il avait un agent sur place donnant les ordres d'opérations au groupe Fouad Saleh, cet agent s'appelant Vahid Gordji. Ce que l'on a appelé l'"affaire Gordji" doit être resituée dans ce cadre 
pour comprendre comment, de dérapages en dérapages, la France et l'Iran ont abouti à la rupture des relations diplomatiques ${ }^{29}$. Au départ, le juge Boulouque s'interroge sur les liens qui unissent Ali Fouad Saleh et Mohamed Mouhajer. Ce dernier, qui a hébergé chez lui Fouad Saleh de novembre 1985 à janvier 1986, est-il lié aux attentats ? A priori, Mouhajer ne fait que de la propagande religieuse, mais il a été, lui aussi, à Qom, il a aidé à plusieurs reprises Fouad Saleh et est très lié au Hezbollah dont son frère est un des trésoriers. La DST commence donc à enquêter plus sérieusement sur Mouhajer, et découvre qu'il est en liaison constante avec un attaché d'ambassade, Vahid Gordji, officiellement simple traducteur, mais officieusement numéro deux de l'ambassade d'Iran, chargé par l'Iran de négocier avec les diplomates français dans l'affaire des otages. De plus, on découvre que Mouhajer est parti en RFA pour acheter une BMW gris foncé au nom de Vahid Gordji début septembre 1986. Or, certains témoins de l'attentat de la rue de Rennes, dont Mme Besse, affirment avoir vu une BMW noire suspecte démarrer en trombe après l'attentat. Circonstance aggravante, l'enquête révèle que Vahid Gordji a fait repeindre sa BMW en couleur claire fin septembre 1986 par Mohamed Moussaoui (petit neveu de Hussein Moussaoui, un des principaux responsables iraniens) qui s'est enfui peu après de France et, sous une fausse identité, a réclamé l'asile politique à la Suisse. C'en est assez pour que le juge Boulouque décide d'entendre Vahid Gordji comme témoin et demande son interpellation. Seulement, cette interpellation gêne considérablement les négociations sur les otages et, selon Charles Villeneuve, des représentants du ministère de l'Intérieur font savoir au juge qu'il serait malséant de l'interroger. Lorsque, le 2 juin 1987, les enquêteurs envoyés par le juge arrivent au domicile de Vahid Gordji, celui-ci n'y est plus. On saura plus tard qu'il s'est réfugié à l'intérieur de l'ambassade pour y bénéficier de l'extra-territorialité. Mais pour l'instant, on ne sait où il a disparu et l'on constate seulement un vent de panique à l'ambassade d'Iran où plusieurs personnes qui, comme Vahid Gordji, ne sont pas des diplomates professionnels, et donc ne disposent pas de l'immunité diplomatique, s'enfuient pour gagner la Suisse. Les services français, interloqués par cette effervescence, supposent qu'ils ont effectivement trouvé les coupables iraniens et que ces derniers prêtent aux enquêteurs plus d'indices qu'ils n'en ont réellement. Charles Pasqua et Robert Pandraud, alertés, exigent que les Iraniens en fuite soient interpellés et raccompagnés jusqu'à leur ambassade en attendant plus ample information. L'ambassade est alors mise sous surveillance discrète, puis de manière publique et médiatique le 28 juin pour faire monter la pression. Le 2 juillet, furieux de cette mise en scène et de cette surveillance, les Iraniens font une conférence de presse où, coup de théâtre, Vahid Gordji réapparaît en tenant son rôle habituel de traducteur et parlant de lui à la troisième personne. Au cours de cette conférence de presse le responsable de l'ambassade, M. Hadadi, affirme que le diplomate Didier Destremeau a prévenu Vahid Gordji, ce qui lui a permis de regagner l'ambassade avant l'interpellation. Toute la presse s'empare alors de l'affaire et la traite sous deux angles : l'implication iranienne dans les attentats et les rivalités de services ou de ministères qui ont abouti à laisser partir Gordji. La situation devient alors inextricable. Le juge Boulouque exige que l'on renforce la surveillance de l'ambassade pour que Vahid Gordji ne puisse s'échapper. Le gouvernement ${ }^{30}$, maintenant que les médias sont au courant, ne peut qu'abonder dans son sens, et désavouer officieusement Didier Destremeau qui nie énergiquement avoir prévenu Gordji mais sans être $\mathrm{cru}^{31}$. Les Iraniens, qui ont voulu jouer des médias contre le gouvernement français en créant la zizanie, sont pris au piège de l'escalade. Comme le gouvernement français ne relâche pas le siège de 
l'ambassade, et remet en cause la normalisation des relations qui passe par une limitation des ventes d'armes à l'Irak le 7 juillet, les Iraniens prennent en otage des diplomates français à Téhéran, et accusent, le 14 juillet, notre Premier secrétaire d'ambassade, M. Torry, d'espionnage, afin de procéder à un marchandage devant aboutir à la libération de Gordji. Seulement, le gouvernement français, loin de l'accepter, refuse l'échange entre M. Torry et Vahid Gordji, car ce serait reconnaître la légitimité des prises d'otages de Français au Liban pour obtenir la libération d'individus jugés coupables par les tribunaux français ${ }^{32}$. La stratégie d'escalade continue donc avec l'envoi de la flotte française dans le Golfe et des attaques iraniennes contre un navire français, le "Ville d'Anvers", puis elle atteint son point culminant avec la rupture des relations diplomatiques, le 17 juillet. Seulement, durant ce temps, l'enquête judiciaire s'est poursuivie et les charges retenues contre Mouhajer et Gordji s'effondrent une à une. Tout d'abord Gordji, qui avait été soupçonné d'être le scripteur des textes de revendication du CSPPA, se voit innocenté par les graphologues. Ensuite, la BMW achetée en RFA ne semble pas être du même modèle que celle décrite par les témoins de la rue de Rennes. Le dossier judiciaire est alors quasiment vide. Même si de très nombreux points obscurs subsistent, il est impossible d'inculper Gordji. En revanche, il est nécessaire de l'entendre comme témoin. Le juge Boulouque, ayant refusé auparavant de l'entendre en se déplaçant dans les locaux de l'ambassade d'Iran ou du Pakistan, la seule solution possible est d'attendre sa sortie, de l'entendre puis de le relaxer, ce qui sera fait lors du dénouement de l'affaire avec les remous que l'on connaît, le juge Boulouque passant pour avoir plié devant le pouvoir politique alors qu'il avait des preuves dans son dossier ${ }^{33}$, bien qu'en réalité il n'avait rien de concret sur le plan judiciaire. Si le juge n'a pas grand chose à se reprocher, il reste une question délicate. A partir de quand le gouvernement fut-il au courant que Gordji était hors de cause judiciairement? Et pourquoi dans ces conditions a-t-il continué la stratégie de l'escalade au lieu de celle de la négociation qu'attendaient les Iraniens? Il semble que Charles Pasqua, voyant l'affolement des Iraniens, ait supposé que Vahid Gordji était plus important qu'il n'y paraissait, et qu'il avait peut-être d'autres choses à se reprocher en dehors des attentats ${ }^{34}$, par exemple l'élimination de moudjahidin du peuple de Radjavi l'opposant au régime de Khomeiny. Il décida alors, même après le 10 août, date à laquelle le juge Boulouque lui a fait part du dossier, de laisser les Iraniens dans l'incertitude en leur faisant croire que les charges contre Gordji étaient irréfutables, afin de s'en servir comme monnaie d'échange contre nos otages détenus au Liban: Gordji, sinon innocent du moins non coupable aux yeux de la justice contre nos otages. Le marché était, somme toute, moins déséquilibré que celui réclamant la libération de Naccache, véritable criminel condamné par la justice. Les Iraniens, inquiets pour le sort de Gordji et prêts à penser que le gouvernement français irait jusqu'à fabriquer de fausses preuves pour le condamner et impliquer l'Iran, fit alors pression sur le Hezbollah pour qu'il libère au moins deux otages: Auque et Normandin. Ces derniers étaient libérés le 28 novembre. Le lendemain, Vahid Gordji était entendu par le juge Boulouque pendant deux heures, et prenait directement l'avion pour Téhéran. Les médias, persuadés de la culpabilité de Gordji, ne savaient s'il fallait louer ou blâmer Charles Pasqua de la libération de deux otages dans de telles conditions. Ils se retournaient donc contre les juges, affirmant que l'affaire Gordji gommait la fermeté de la condamnation d'Abdallah ${ }^{35}$. Finalement, il résulte de ces événements qu'il est impossible judiciairement de prouver l'implication directe de l'Iran dans les attentats bien que certains indices puissent conduire logiquement à eux. En effet, si les Iraniens 
n'ont pas directement commandité de Paris les attentats par l'intermédiaire de Gordji, il n'est pas impossible que certains d'entre eux (Rafigh Doust) en soient les commanditaires indirects, en dehors de tout rapport avec Khomeiny, par Hezbollah interposé, et à titre d'initiative personnelle. Simplement il est quasiment certain qu'ils n'ont pas fourni d'aide matérielle et que toute l'infrastructure logistique est venue du Hezbollah lui-même, ce qui explique les faiblesses et l'originalité des modalités de transfert choisies. De plus l'accumulation matérielle de preuves laissées en évidence dans les divers domiciles témoigne d'un amateurisme total. Cet amateurisme tranche lui aussi avec l'image d'un groupe de professionnels bien organisé, relais d'une puissance étrangère agissant sur le territoire français. Il met à mal les visions géostratégiques globales qui raisonnent sur le terrorisme comme pour les actions de services secrets et n'arrivent pas à penser le terrorisme comme logique de violence politique transnationale incluant des acteurs de niveaux hiérarchiques différents, caractérisés par leur asymétrie mais fortement impliqués dans leur totalité dans le jeu politique. C'est ce vecteur de transnationalité qui est sans doute le plus original et le plus nouveau dans la relation terroriste incluant des acteurs extérieurs qui parrainent une organisation écran. A ce titre, il nous faut maintenant essayer de tirer les leçons des attentats de 1986 et reconstruire intellectuellement la configuration des positions des acteurs au sein de la relation terroriste en analysant leurs motivations et leur coups tactiques $^{36}$

\section{NOTES}

1. Certains de nos collègues succombent malgré tout à la tentation de la notoriété facile, légitimant ainsi le soupçon des autres. Pour plus de détails sur les conditions de production du savoir universitaire à propos du terrorisme, voir notre article "Terrorisme et antiterrorisme en France", Cahiers de la Sécurité Intérieure (1), avriljuin 1990.

2. Pour une approche théorique du terrorisme analysant sa spécificité à l'égard d'autres formes de violence, les mécanismes qui structurent les comportements des acteurs, les stratégies de ces derniers et l'obligation d'avoir une analyse relationnelle insistant sur l'échange des coups tactiques... voir Didier Bigo, Daniel Hermant, La relation terroriste, Etudes Polémologiques (47), 1988, 192 pages

3. Nous avons pu obtenir dans le cadre d'un contrat de recherche avec le ministère de la Recherche et l'Institut des hautes études de sécurité intérieure les deux réquisitoires du procès correctionnel du 9 mars 1990 et du procès criminel qui devrait se tenir l'année prochaine. Nous avons aussi depuis 1986 rencontré de nombreux responsables : Les juges Boulouque, Fourvel et Marsaud, les responsables de l'Unité de coordination et de liaison antiterroriste (UCLAT) MM. Querry et Tchividjian, des responsables du Quai d'Orsay, certains des intermédiaires choisis par les hommes politiques pour les négociations à propos des otages et des attentas de 86, à quoi il faut ajouter les livres témoignages de certains d'entre eux comme celui de Daniel Burdan, Neuf ans à la division antiterroriste, Paris : Laffont, 1990, ou les récits des otages... 
4. Commis par Habib Mohamar qui se réclamera de l'organisation Abou Ibrahim, groupe du 15 mai, non revendiqué à l'époque

5. Certains enquêteurs font remonter la série à l'attentat du 9 mars dont s'occupe le juge Rivière, ce qui rendrait les attentats contemporains des prises d'otages au Liban. 6. Le foyer installé dans le 13ème arrondissement de Paris sera fermé en 1984, réouvert au Kremlin Bicêtre, dans la banlieue parisienne et définitivement interdit par Gilles Boulouque en 1987.

7. Ce qui est possible, mais n'empêche pas l'irritation des auteurs parisiens des attentats et la relance immédiate de ceux-ci au lendemain de l'interpellation et de la relaxe de certains d'entre eux ; relaxe qui renforce leur sentiment d'être protégés, d'avoir la baraka, bref d'être quasi-invulnérables, Cela pose la question cruciale des liens exacts entre les auteurs des prises d'otages et les attentats parisiens.

8. Il s'agit ici de la vision des Abdallah car faut-il le rappeler en ces temps de marchandage généralisé sous l'égide de l'ONU et en contradiction avec les déclarations des gouvernements depuis près de dix ans, rien ne justifie d'échanger un otage contre un responsable d'attentats.

9. Certains éléments troublants expliquent cet entêtement sur la piste Abdallah. Tout d'abord il est demandé sa libération sous son nom d'Abdel Kader Essaadi, Nabil Dagher appartenait au FARL, Jean-Paul Mazurier, avocat d'Abdallah renseignant la DST, affirme avoir vu chez son client une lettre du CSPPA, des témoins reconnaissent la photo d'Emile Abdallah (en fait Habib Haïdar qui lui ressemble fortement pour un oeil non exercé). Enfin et surtout, les services syriens et algériens correspondants de la DST persuadent celle-ci que les Abdallah sont bien les coupables et se posent en médiateurs pour en tirer Profit

10. D'autant que, simultanément, les services français démembraient discrètement une filière d'explosifs qui passait par la Côte d'Ivoire : sur ce sujet voir Didier Bigo,

"l'émigration libanaise en Côte d'Ivoire : la stigmatisation d'une diaspora", Cahiers de l'Orient, janvier 1992.

11. Sur les fausses pistes, voir Didier Bigo, Daniel Hermant, "Tiers, médiateurs et parasites", Etudes Polémologiques (49)

12. $D$ 'après certains entretiens, on peut supposer que le plan iranien en question était plus global. Il visait avant tout à récupérer, voire à racheter, les otages français au Liban afin de faire monter la pression dans les négociations en étant sûr de pouvoir les libérer par la suite en fonction des besoins. Il faisait l'analyse de l'aide française à l'Irak et tout en citant les attentats, n'indiquait pas explicitement que l'Iran les avait commandité au Hezbollah ou même qu'il aurait fourni une aide financière ou logistique sur place, mais prend-on le risque d'écrire de telles choses?

13. Mouhajer sera relâché.

14. Le groupe Hamade se compose de Mohamed Ali, de son frère Abbas,...

15. Bundeskriminalamt dont un service coordonne la lutte anti-terroriste au niveau fédéral

16. Il aurait parlé de prendre les armes avec les Kurdes contre les hypocrites du régime iranien accusant les mollah de "proxénétisme à la suite des "remariages" des veuves de guerre.

17. Le 20 mars 1985, Fouad Saleh, sans argent, est expulsé de son logement et demande à Badaoui de l'héberger

18. Il ne s'agit pas non plus d'un mercenariat. Ali Fouad Saleh n'a pas de domicile fixe, pas d'argent... ses compagnons non plus. Au total, ils auraient touché moins de vingt 
mille francs de Beyrouth à eux tous pour faire face à leurs dépenses. Nous sommes loin des hôtels de luxe dans lesquels se complaisait parait-il Carlos! Quant aux stupéfiants que l'on retrouve, ils n'en consomment pas, dès lors ils avaient peut-être le désir de les vendre mais n'ont pas eu le temps de le faire. Ils servaient sans doute d'intermédiaire pour le trafic de drogue des familles Hamade et Gosn.

19. Dans Etudes Polémologiques, nous avions mis en garde contre les dangers d'amalgame, mais, d'une certaine manière, nous étions resté trop prêt de ces thèses en reprenant l'idée douteuse de "communauté relais"

20. Voir Rémy Leveau, "le non-passage au terrorisme de l'immigration maghrébine", Etudes Polémologiques (49)

21. La DST soupçonnera un membre du FPLP/CG d'être le fameux scripteur des revendications du CPPA après avoir abandonné la piste Abdallah

22. Numéro que l'on retrouva aussi dans le carnet d'Hamade.

23. A titre d'hypothèse, on peut penser qu'au mois de mars les explosifs ne sont plus suffisants pour lancer une nouvelle campagne, ce qui expliquerait le voyage de Saleh en juin et la programmation pour septembre des attentats, sans négliger la possibilité d'une certaine malignité qui pousse à faire croire à la piste Abdallah

24. Frère aîné de Mohamed et Abbas Hamade.

25. Voir plus haut, l'arrestation par les Allemands des frères Hamade.

26. Pour une analyse des relations entre les groupes Hamade et Fouad Saleh ainsi que pour l'analyse du traitement judiciaire différencié entre les deux pays, voir Sylvie Lemasson et Didier Bigo : article à paraître.

27. Une société en liquidation judiciaire choisie pour des raisons de liens familiaux, le stockage dans des sociétés tierces par perte de la LTA, l'absence d'argent qui éveillera les soupçons des personnes de la société Defie et aidera à l'identification...plus bien sûr tous les ratés techniques des attentats, et un chef de réseau à la rue et dans divers logements de fortune.

28. Voir l'article de Michel Wieviorka dans ce même numéro. Comme il le dit justement cette thèse, même "revisitée" après les déclarations des responsables des services de l'Est, reste inopérante pour expliquer le terrorisme en grande partie parce qu'elle le conçoit dans le cadre d'une stratégie indirecte de guerre froide et ne tient pas compte de l'autonomie des acteurs libres de souveraineté. De plus les déclarations récentes au journal Spiegel sur la collaboration de la Stasi et de la RAF outre qu'elles portent sur une période où le sigle RAF a été utilisé par des groupuscules de quelques personnes isolées de la direction (pour échapper aux investigations et recoupements informatiques du BKA), restent sujettes à caution. Faites dans le but de récupérer Honnecker, réfugié en URSS, elles servaient un projet politique précis et permettaient en même temps aux "responsables des servires secrets" des pays de l'Est de vendre souvent très cher leurs soi-disant révélations. Quand a lieu la "désinformation" : avant ou maintenant ? la RFA n'était-elle pas d'accord avec la porte de sortie du terrorisme ouverte à des membres de la RAF qui "prenaient leur retraite" (et ne continuaient pas la lutte armée depuis un sanctuaire) en RDA. L'exemple italien nous apprend que les services occidentaux savent aussi manier la désinformation et il est encore hasardeux de tirer des conclusions de ces "révélations".

29. Ce qui, pour la France, ne s'est produit que dans des cas rarissimes, tous liés à des situations de conflit mondial.

30. Qui depuis le 18 juin ou même le 2 savait que Gordji était à l'ambassade et avait commencé des tractations. 
31. Didier Destremeau a-t-il agi de sa propre initiative ? C'est peu probable. Alors à quel niveau de responsabilité se situe-t-elle? Son chef de service, son ministre, le gouvernement? Autant d'hypothèses ouvertes même si celle du ministre est la plus crédible.

32. De plus Torry, prévenu des risques, a pris ses précautions et a d'amples provisions dans l'ambassade, ce qui n'est pas le cas des Iraniens à Paris dont on a coupé l'eau, l'électricité...

33. Voir la caricature de Plantu dans le journal Le Monde où Gilles Boulouque se transforme en stewart et propose à Gordji : "fumeur" ou "non fumeur".

34. Ce qui expliquerait la panique de l'ambassade

35. Double erreur d'une presse le plus souvent mal renseignée.

36. Compte tenu de la place limitée, nous nous concentrerons sur les organisations écrans et leurs parrains mais une analyse complète nécessite de prendre en compte l'attitude des pouvoirs publics français, leurs stratégies, leurs contradictions, les coups qu'ils jouent dans l'arène politique compliquée par la cohabitation et les positions des "Tiers", des victimes, de la population française, de la communauté immigrée... Sur tous ces point, voir Etudes Polémologiques (49) dont la seconde partie de notre article s'inspire.

\section{RÉSUMÉS}

Les attentats qui ont frappé la France en 1985/86 sont mal connus. Les médias, les hommes politiques en ont parlé sur le moment et ont ensuite évité de revenir sur les erreurs qu'ils avaient commises. Grâce au procès criminel du groupe Ali Fouad Saleh et grâce à de nombreux entretiens avec des responsables des services, l'auteur revient sur le déroulement exact des attentats et sur les preuves judiciaires impliquant les membres des organisations clandestines. Dans une deuxième partie il évoque les différente hypothèses possibles concernant la participation du Hezbollah et des Iraniens. Au-delà, il propose un schéma d'analyse qui remet en cause les visions du terrorisme en terme de " réseau de la terreur ", " stratégies indirectes d'Etats " et insiste sur la transnationalié des transferts de violence qui ont caractérisé ces attentats. A partir de ce cas d'espèce, c'est une autre théorie du " terrorisme " dit international qui est proposé.

The terrorist bombings that rocked France during 1985-1986 are not well known. The media and the politicians spoke of them at the time, but preferred not to refer to them later so as not to draw attention to their mistakes at the time. The author provides a detailed description of the events and the legal evidence implicating members of secret organisations on the basis of the court proceedings of the prosecution of Ali Fouad Saleh and his group, and numerous interviews with the officials concerned. In a second part, he evokes the different hypotheses concerning the participation of Hezbollah and the Iranians. Moreover, he presents an analysis that questions such views of terrorism as those based on "terror networks " or " indirect governmental strategies ". Rather he sees the causes in a transnational transfer of violence. This leads to a new transnational theory which should abandon the overtly simple concept of international terrorism. 
INDEX

Index géographique : France

Mots-clés : attentat, réseaux transnationaux, sociologie des conflits (polémologie), violence Index chronologique : 1980 - 1990

\section{AUTEUR}

DIDIER BIGO

Maître de conférences des Universités à l'Institut d'Etudes Politiques de Paris, rédacteur en Chef de Cultures \& Conflits 